\title{
A MULTISCALE FINITE ELEMENT METHOD FOR PARTIAL DIFFERENTIAL EQUATIONS POSED IN DOMAINS WITH ROUGH BOUNDARIES
}

\author{
ALEXANDRE L. MADUREIRA
}

\begin{abstract}
We propose and analyze a finite element scheme of multiscale type to deal with elliptic partial differential equations posed in domains with rough boundaries. There is no need to assume that the boundary is periodic in any sense, so the method is quite general. On the other hand, if the boundary is periodic we prove convergence of the scheme.
\end{abstract}

\section{INTRODUCTION}

Several applications require solving PDEs in domains with rough boundaries. Finding exact solutions is in general out of question, and numerically approximating the problem can be hard since discretizing the boundaries requires a sophisticated and quite refined, i.e. expensive, mesh. That is because good quality meshes for such domains often over-refine unnecessarily the interior of the domain.

Assuming that the wrinkles of the boundary are, in a proper sense, periodic, several authors proposed effective boundary conditions, also known as wall laws, on a mollified boundary that somehow upscaled the small scale geometry $1-6$, 8 10, 12, 14, 25, 26. This was possible for several operators and geometries, and involved solving cell problems, as is typical in homogenization procedures. Also, the article [13] recently presented the case of random, spatially homogeneous wrinkles.

Despite the remarkable advances just mentioned, to the best of our knowledge, the case of nonperiodic wrinkles was left untouched, with the exception noted above.

On the other hand, some numerical methods for PDEs with oscillatory coefficients were recently suggested and analyzed [11,16, 24,30]. A desirable feature of these numerical schemes is that there is no need to derive a homogeneous equation, and only then discretize. On the contrary, the methods tackle the problems in their original forms.

The goal of the present paper is to propose a Multiscale Finite Element Method (MsFEM) to numerically solve PDEs in domains with oscillatory boundaries.

Consider the sequence of problems parameterized by $\varepsilon<1$ : find $u^{\varepsilon}$ such that

$$
\begin{gathered}
-\Delta u^{\varepsilon}=f \quad \text { in } \Omega^{\varepsilon}, \\
u^{\varepsilon}=0 \quad \text { on } \partial \Omega^{\varepsilon},
\end{gathered}
$$

Received by the editor February 12, 2007 and, in revised form, October 5, 2007.

2000 Mathematics Subject Classification. Primary 35J05, 35J25, 65N12, 65N15, 65N30.

The author was partially supported by the CNPq/Brazil Projects 306104/2004-0 and 486026/2006-0, and also by FAPERJ Project APQ1 E-26/170.629/2006.

(C)2008 American Mathematical Society Reverts to public domain 28 years from publication 


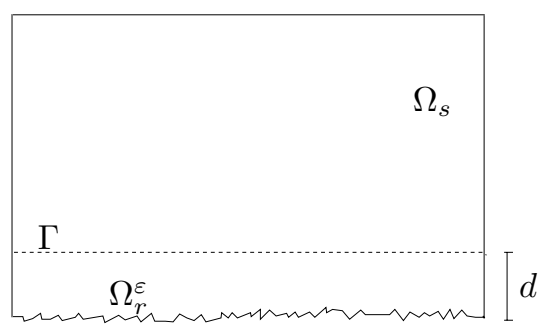

Figure 1. The domain $\Omega^{\varepsilon}$.

for $f \in L^{2}\left(\Omega^{\varepsilon}\right)$. For simplicity, we assume that the domain $\Omega^{\varepsilon}$ is a rectangle with a rough bottom, as depicted in Figure 1. For $d>\varepsilon$, let

$$
\Omega^{\varepsilon}=\left\{\mathbf{x}=(x, y) \in \mathbb{R}^{2}: 0<x<1,-d+\psi_{r}^{\varepsilon}(x)<y<1\right\},
$$

where the Lipschitz function $\psi_{r}^{\varepsilon}$ is such that $\psi_{r}^{\varepsilon}(0)=\psi_{r}^{\varepsilon}(1)=0$, and $\left\|\psi_{r}^{\varepsilon}\right\|_{L^{\infty}(0,1)}<$ $\varepsilon$. The graph of $\psi_{r}^{\varepsilon}$ defines $\Gamma_{r}^{\varepsilon}$, the lower boundary of $\Omega^{\varepsilon}$ :

$$
\Gamma_{r}^{\varepsilon}=\left\{(x, y) \in \partial \Omega^{\varepsilon}: y=-d+\psi_{r}^{\varepsilon}(x)\right\} .
$$

For the sake of the forthcoming analysis, it is convenient to consider $\Omega^{\varepsilon}$ as the union of an $\varepsilon$-independent domain and a "rough rectangle"; see (2.1) below. The parameter $d$ indicates the "thickness" (disregarding the wrinkles) of the rugged part.

Next, we outline the contents of this paper. In Section 2 we introduce a numerical scheme to solve (1.1), and in Section 3. we do an error analysis for the periodic case.

We now briefly introduce and explain some basic notation that we use throughout this paper. As usual, if $D$ is an open set, then $L^{2}(D)$ is the set of square integrable functions in $D$, and $H^{s}(D)$ is the corresponding Sobolev space of order $s$, for a real number $s$. We denote the norms of those spaces by $\|\cdot\|_{L^{2}(D)}$ and $\|\cdot\|_{H^{s}(D)}$. Without loss of generality, we have chosen to work in two dimensions. Nonetheless, all that follows can be generalized to the three-dimensional case. Bold fonts indicate twodimensional vectors, and we denote by $c$ a generic constant (not necessarily the same in all occurrences) which is independent of $\varepsilon$, but may depend on Sobolev norms of $f$.

\section{Multiscale finite Element method}

We propose in this section a multiscale numerical method to approximate the solutions of (1.1). Variants of this method were successfully employed in different contexts, and we extend their use to the present problem.

In the MsFEM [19 24, the basis functions are local (elementwise) solutions of the original problem, and should capture the local features of the problem to upscale this information into the global formulation.

In our setting, we propose a Galerkin method where the basis functions in the trial and test spaces have local support, as in the traditional finite element method. Nevertheless, the present method is unusual since it does not use polygonal shape functions. Actually, the elements are not even triangles or quadrilaterals, but have a "rough geometry" whenever they intersect the rough boundary. The basic idea behind the method is that the influence of the geometry should be captured by the 

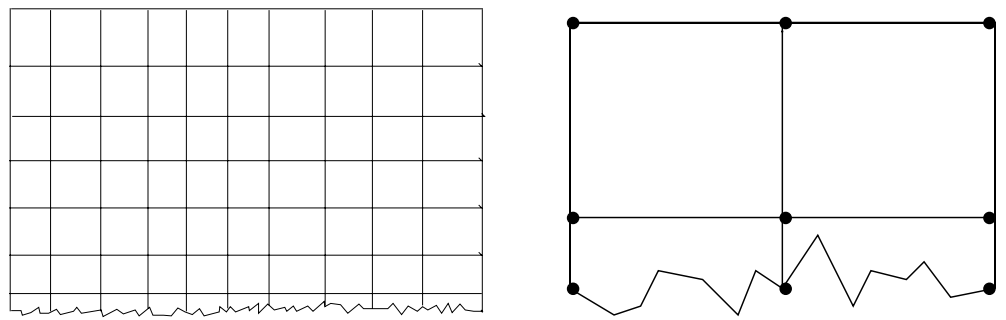

Figure 2. A mesh for $\Omega^{\varepsilon}$, and a patch of elements of $\mathcal{T}_{h}$ intercepting $\Gamma_{r}^{\varepsilon}$.

basis functions without affecting the dimension of the finite element space. So our multiscale method is of Galerkin type, where the basis functions are local solutions of the operator under consideration (Laplacian), possibly in a rough element.

Before discretizing $\Omega^{\varepsilon}$ in finite elements, we define

$$
\Omega_{s}=(0,1) \times(0,1), \quad \Omega_{r}^{\varepsilon}=\Omega^{\varepsilon} \backslash \overline{\Omega_{s}}, \quad \Gamma=(0,1) \times\{0\} .
$$

Note that

$$
\Omega_{r}^{\varepsilon}=\left\{\mathbf{x}=(x, y) \in \Omega^{\varepsilon}: 0<x<1,-d+\psi_{r}^{\varepsilon}(x)<y<0\right\} .
$$

Let $N \in \mathbb{N}$ and $h=1 /(N+1)>\varepsilon$. For $i=0, \ldots, N$, let $K_{i}^{\varepsilon}=\{(x, y) \in$ $\left.\Omega_{r}^{\varepsilon}: i h<x<(i+1) h\right\}$. Note that such elements define a tiling of $\Omega_{r}^{\varepsilon}$. Next, we introduce a Cartesian mesh for $\Omega_{s}$ using squares of side $h$. Such a procedure induces a partition $\mathcal{T}_{h}$ of $\Omega^{\varepsilon}$ in finite elements, not all of them being squares. Indeed, if $K \in \mathcal{T}_{h} \cap \Omega_{s}$, then $K$ is a square of size $h$. Otherwise, $K$ has a rough bottom, and straight lateral and top edges; see Figure 2. The mesh $\mathcal{T}_{h}$ has as nodal points the set $\mathcal{N}=\left\{(i h, j h) \in \Omega^{\varepsilon}: i=1, \ldots, N, j=0, \ldots, N\right\}$.

For each node $\mathbf{x}_{i} \in \mathcal{N} \cap \Omega_{s}$, we associate a piecewise bilinear polynomial $\lambda_{i} \in$ $H_{0}^{1}\left(\Omega^{\varepsilon}\right)$ such that $\lambda_{i}\left(\mathbf{x}_{j}\right)=\delta_{i j}$ for all $\mathbf{x}_{j} \in \mathcal{N}$.

For each node $\mathbf{x}_{i} \in \mathcal{N} \cap \Gamma$, we define $\lambda_{i} \in H_{0}^{1}\left(\Omega^{\varepsilon}\right)$ such that

$$
-\Delta \lambda_{i}=0 \quad \text { in } \bigcup_{j=0}^{N} K_{j}^{\varepsilon},
$$

$$
\lambda_{i}\left(\mathbf{x}_{j}\right)=\delta_{i j} \quad \text { for all } \mathbf{x}_{j} \in \mathcal{N}, \quad \lambda_{i} \text { is linear on } \bigcup_{j=0}^{N} \partial K_{j}^{\varepsilon} \cap \Omega^{\varepsilon} .
$$

We extend $\lambda_{i}$ to $\Omega_{s}$ by imposing that $\lambda_{i}$ is piecewise bilinear in $\Omega_{s}$.

Using the functions just defined, we set

$$
V_{h}^{\varepsilon}=\operatorname{span}\left\{\lambda_{i}\right\} \subset H_{0}^{1}\left(\Omega^{\varepsilon}\right) .
$$

The MsFEM solution $u_{h}^{\varepsilon} \in V_{h}^{\varepsilon}$ is simply the Galerkin approximation of $u^{\varepsilon}$ in $V_{h}^{\varepsilon}$, i.e,

$$
\int_{\Omega^{\varepsilon}} \nabla u_{h}^{\varepsilon}(\mathbf{x}) \cdot \nabla v_{h}(\mathbf{x}) d \mathbf{x}=\int_{\Omega^{\varepsilon}} f(\mathbf{x}) v_{h}(\mathbf{x}) d \mathbf{x} \quad \text { for all } v_{h} \in V_{h}^{\varepsilon} .
$$

Remark 2.1. Note that the computation of each basis function is $\varepsilon$-dependent, thus expensive. Although the cost can be mitigated by parallelization, this is clearly a drawback. Nonetheless, as noted by Marcus Sarkis [31, at least for two-dimensional 
problems, it is possible to obtain a quite narrow banded stiffness matrix by numbering the nodes properly.

Remark 2.2. The present method is particularly attractive if the problem (1.1) is to be solved repeatedly for different source terms. Indeed, the basis functions need to be computed only once for a given geometry, and the size of the stiffness matrix is $\varepsilon$-independent.

\section{Numerical ANALYSis}

In this section we perform the error analysis for the numerical schemes proposed. Such analysis is based on asymptotic methods, and we restrict ourselves to the case of periodic wrinkles, when the solution has a well-known structure. We do not present the arguments leading to the development of the terms in the expansion, but we rather simply define the terms. Madureira and Valentin [25,26] consider a complete asymptotics for a curved rough boundary.

By periodic wrinkles, we mean that $\psi_{r}^{\varepsilon}(x)=\varepsilon \psi_{r}(x / \varepsilon)$, where $\psi_{r}$ is periodic with period 1 , and $\left\|\psi_{r}\right\|_{L^{\infty}(\mathbb{R})}<1$. In what follows, we assume for simplicity that $f$ vanishes in $\Omega_{r}^{\varepsilon}$. We also assume that there exist constants $\gamma \in(0,1]$, and $c_{0}$ positive such that

$$
c_{0} \varepsilon^{1-\gamma} \leq d,
$$

where we recall that the parameter $d$ helps to define the rough domain $\Omega_{r}^{\varepsilon}$. See Figure 1, and equations (1.2), (2.2).

Remark 3.1. The restriction (3.1) indicates that $d$ cannot be "too small". Indeed, the rough boundary originates ripples in $u^{\varepsilon}$ that decay exponentially as $y / \varepsilon$ grows, and it is important in the numerical analysis that the oscillatory part of the solution in $\Omega_{s}$ is at most polynomially small with respect to $\varepsilon$. So, the distance $d$ helps to define the region $\Omega_{s}$ which is free from the high-gradient part of the solution.

3.1. Asymptotic expansion for the exact solution. To make clear how the solution of equation (1.1) depends on the small parameter $\varepsilon$, we expand $u^{\varepsilon}$ in a formal power series with respect to $\varepsilon$.

We develop the expansion using the decomposition (2.1) of $\Omega$ in $\Omega_{r}^{\varepsilon}$ and $\Omega_{s}$, and the expansions are coupled by boundary conditions on $\Gamma$. In $\Omega_{s}$ the asymptotic expansion of $u^{\varepsilon}$ is composed by smooth terms, and in $\Omega_{r}^{\varepsilon}$ the expansion is made up of a highly oscillatory part which decays exponentially to zero away from $\Gamma_{r}^{\varepsilon}$, plus a linear function in $y$. The first few terms of the expansion are as follows:

$$
u^{\varepsilon}(\mathbf{x}) \sim \begin{cases}(d+y-\varepsilon W-\varepsilon z) \frac{\partial u^{0}}{\partial y}(x, 0)+(d+y) \frac{\partial u^{1}}{\partial y}(x, 0)+\ldots & \text { in } \Omega_{r}^{\varepsilon}, \\ u^{0}(\mathbf{x})+u^{1}(\mathbf{x})+\ldots & \text { in } \Omega_{s} .\end{cases}
$$

The first term of the asymptotics solves

$$
\begin{aligned}
& -\Delta u^{0}=f \quad \text { in } \Omega_{s}, \\
& u^{0}=0 \quad \text { on } \partial \Omega_{s}, \quad u^{0}=0 \quad \text { on } \Omega_{r}^{\varepsilon} \text {. }
\end{aligned}
$$

Remark 3.2. In (3.2) and the remainder of this paper, the term $\partial u^{0} / \partial y(x, 0)$ denotes the restriction of $\partial u^{0} /\left.\partial y\right|_{\Omega_{s}}$ on $\Gamma$. The same convention holds for $\partial u^{1} / \partial y(x, 0)$, etc. 


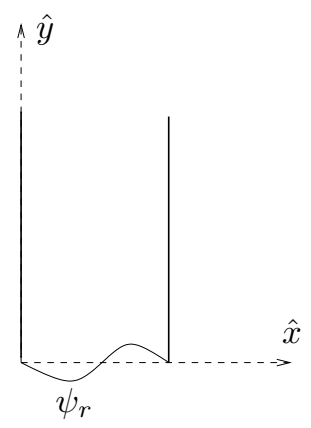

Figure 3. The cell domain.

To continue the description of the expansion, it is necessary to introduce a cell problem. This is no different from other singularly perturbed problems, perhaps elliptic PDEs with highly oscillatory coefficients being the most notorious. Such cell problems are an essential part in up-scaling procedures, bringing information related to the small scale geometry into the large scale behavior of the solution.

In the present case, the cell problem is defined in the semi-infinite strip $\Omega_{r}$, which "contains" the geometry of the wrinkles,

$$
\Omega_{r}=\left\{(\hat{x}, \hat{y}) \in \mathbb{R}^{2}: \hat{x} \in(0,1), \hat{y}>\psi_{r}(\hat{x})\right\},
$$

i.e., $\Omega_{r}$ occupies the region delimited by straight lateral boundaries at $\hat{x}=0$ and $\hat{x}=1$, and by the lower boundary $\Gamma_{r}=\left\{\left(\hat{x}, \psi_{r}(\hat{x})\right) \in \mathbb{R}^{2}: \hat{x} \in(0,1)\right\}$; see Figure 3 ,

We define $C_{\text {per }}^{\infty}\left(\Omega_{r}\right)$ by restricting to $\Omega_{r}$ the functions in $C^{\infty}\left(\mathbb{R}^{2}\right)$ which are oneperiodic with respect to $\hat{\theta}$. Let $H_{\text {per }}^{1}\left(\Omega_{r}\right)$ be the closure of $C_{\text {per }}^{\infty}\left(\Omega_{r}\right)$ with respect to the $H^{1}\left(\Omega_{r}\right)$ norm. We also introduce the space of exponentially decaying functions

$$
S\left(\Omega_{r}\right)=\left\{w \in H_{\text {per }}^{1}\left(\Omega_{r}\right): w(\hat{x}, \hat{y}) e^{\alpha \hat{y}} \in H^{1}\left(\Omega_{r}\right) \text { for some } \alpha>0\right\} .
$$

It is possible to show [7,26 that there exists unique $w \in S\left(\Omega_{r}\right)$, and a unique constant $z$ such that

$$
\Delta w=0 \quad \text { in } \Omega_{r}, \quad w=\hat{y}-z \quad \text { on } \Gamma_{r} .
$$

Moreover, $0 \leq z \leq\left\|\psi_{r}\right\|_{L^{\infty}(0,1)}$, and $\|w\|_{H^{1}\left(\Omega_{r}\right)} \leq c$, where $c$ depends on the geometry only. Both $z$ and $w$ are related to the boundary layers that naturally occur in the original problem. Let $W(x, y)=w\left(\varepsilon^{-1} x, \varepsilon^{-1}(d+y)\right)$ in $\Omega_{r}^{\varepsilon}$.

Finally, let

$$
\begin{aligned}
-\Delta u^{1} & =0 \quad \text { in } \Omega_{s}, \\
u^{1}=(d-\varepsilon z) \frac{\partial u^{0}}{\partial y} \quad \text { on } \Gamma, \quad u^{1} & =0 \quad \text { on } \partial \Omega_{s} \backslash \Gamma, \quad u^{1}=0 \quad \text { on } \Omega_{r}^{\varepsilon} .
\end{aligned}
$$

Remark 3.3. The above terms are slightly different from what usually appears in the literature. The difference is due to our definition of $d$, since usually $d=d_{0} \varepsilon$, where $d_{0}$ is a predefined constant. See for instance [25,26].

Albeit (3.2) is formal, it is possible to show [26] that if

$$
e(\mathbf{x})=u^{\varepsilon}(\mathbf{x})-u^{0}(\mathbf{x})-u^{1}(\mathbf{x})+(y-\varepsilon W) \frac{\partial u^{0}}{\partial y}(x, 0) \chi_{r}^{\varepsilon},
$$


where $\chi_{r}^{\varepsilon}$ is the characteristic function of $\Omega_{r}^{\varepsilon}$, and under the assumption (3.1), there exists an $\varepsilon$-independent constant $c$ such that

$$
\|e\|_{H^{1}\left(\Omega^{\varepsilon}\right)} \leq c d^{3 / 2}, \quad\|e\|_{H^{1}\left(\Omega_{s}\right)} \leq c d^{2} .
$$

To obtain the above estimates, it is useful to have the following bounds for each individual term. Such bounds follow from classical regularity estimates, and changes of coordinates.

Lemma 3.4. Let $u^{0}, u^{1}$, and $W$ be defined as above. Then there exists an $\varepsilon$ independent constant $c$ such that

$$
\left\|u^{0}\right\|_{H^{1}\left(\Omega_{s}\right)}+d^{-1}\left\|u^{1}\right\|_{H^{1}\left(\Omega_{s}\right)}+\varepsilon^{-1 / 2}\|W\|_{L^{2}\left(\Omega_{r}^{\varepsilon}\right)}+\varepsilon^{1 / 2}\|\nabla W\|_{L^{2}\left(\Omega_{r}^{\varepsilon}\right)} \leq c .
$$

Proof. The estimate for $u^{0}$ follows immediately from (3.3), and (3.5) yields the bound for $u^{1}$. The estimates related to $W$ come from the change of coordinates $\hat{x}=\varepsilon^{-1} x, \hat{y}=\varepsilon^{-1}(d+y)$, since, e.g.,

$$
\int_{\Omega_{r}^{\varepsilon}}|W(\mathbf{x})|^{2} d \mathbf{x}=\varepsilon \int_{\Omega_{r}}|w(\hat{\mathbf{x}})|^{2} d \hat{\mathbf{x}}, \quad \int_{\Omega_{r}^{\varepsilon}}|\nabla W(\mathbf{x})|^{2} d \mathbf{x}=\varepsilon^{-1} \int_{\Omega_{r}}|\nabla w(\hat{\mathbf{x}})|^{2} d \hat{\mathbf{x}} .
$$

3.2. Analysis of the multiscale finite element method. The goal of this subsection is to prove a convergence result for the MsFEM. We first note that Poincaré's inequality holds uniformly with respect to $\varepsilon[26$, i.e., there exists an $\varepsilon$-independent constant such that

$$
\|v\|_{L^{2}\left(\Omega^{\varepsilon}\right)} \leq|v|_{H^{1}\left(\Omega^{\varepsilon}\right)}
$$

for all $v \in H_{0}^{1}\left(\Omega^{\varepsilon}\right)$. With that, we conclude that Céa's Lemma also holds uniformly in $\varepsilon$, as we state below.

Lemma 3.5 (Céa's Lemma). Let $u^{\varepsilon} \in H_{0}^{1}\left(\Omega^{\varepsilon}\right)$ be the weak solution of (1.1), and $u_{h}^{\varepsilon} \in V_{h}^{\varepsilon}$ the solution of (2.4). Then there exists an $\varepsilon$-independent constant $c$ such that

$$
\left\|u^{\varepsilon}-u_{h}^{\varepsilon}\right\|_{H^{1}\left(\Omega^{\varepsilon}\right)} \leq c \inf _{v_{h}^{\varepsilon} \in V_{h}^{\varepsilon}}\left|u^{\varepsilon}-v_{h}^{\varepsilon}\right|_{H^{1}\left(\Omega^{\varepsilon}\right)} .
$$

Hence, the task now is to find a good approximation for $u^{\varepsilon}$ in $V_{h}^{\varepsilon}$. As in [23], we use the asymptotics of both $u^{\varepsilon}$ and the basis functions as a tool. We present here the asymptotic expansion in $K_{i}^{\varepsilon}$ of the basis function $\lambda_{i}$ defined in (2.3), corresponding to a nodal point $(i h, 0) \in \Gamma \cap \mathcal{N}$. The asymptotics of $\lambda_{i}$ in $K_{i-1}^{\varepsilon}$ is similar. In $K_{i}^{\varepsilon}$ we have that

$$
\lambda_{i}(\mathbf{x})=\frac{1}{h(d-\varepsilon z)}\left[(d+y-\varepsilon W-\varepsilon z)\left(x_{i+1}-x\right)+\varepsilon \theta_{i}(\mathbf{x})+\varepsilon r_{i}(\mathbf{x})\right],
$$

where $x_{i+1}=(i+1) h$, and $\theta_{i}$ solves

$$
\begin{aligned}
-\Delta \theta_{i}=0 \quad \text { in } K_{i}^{\varepsilon}, & \\
\theta_{i}(x, y)=\left(x_{i+1}-x\right)\left[W(x, y)-\frac{z y}{d}\right] & \text { on } \partial K_{i}^{\varepsilon} \backslash \Gamma_{r}^{\varepsilon}, \quad \theta_{i}=0 \quad \text { on } \Gamma_{r}^{\varepsilon} .
\end{aligned}
$$

Also,

$$
-\Delta r_{i}=2 \frac{\partial W}{\partial x} \quad \text { in } K_{i}^{\varepsilon}, \quad r_{i}=0 \quad \text { on } \partial K_{i}^{\varepsilon}
$$


Note that the expansion (3.2) in $\Omega_{r}^{\varepsilon}$ and (3.7) are quite similar since in both cases the lower order terms involve the expression $d+y-\varepsilon W-\varepsilon z$ times a function of $x$ only, that is, $\partial u^{0} / \partial y$ in (3.2), and $\left(x_{i+1}-x\right) /[h(d-\varepsilon z)]$ in (3.7).

The following lemma presents an upper bound for the $H^{1}$ norms of $r_{i}$ and $\theta_{i}$.

Lemma 3.6. Let $r_{i}$ and $\theta_{i}$ be defined as above. Then there exists a constant $c$ independent of $\varepsilon$ and $h$ such that

$$
\left\|r_{i}\right\|_{H^{1}\left(K_{i}^{\varepsilon}\right)} \leq c \varepsilon^{1 / 2} h^{1 / 2}, \quad\left\|\theta_{i}\right\|_{H^{1}\left(K_{i}^{\varepsilon}\right)} \leq c h .
$$

Proof. It follows from standard estimates that $\left\|r_{i}\right\|_{H^{1}\left(K_{i}^{\varepsilon}\right)} \leq\|W\|_{L^{2}\left(K_{i}^{\varepsilon}\right)}$. Then, arguing as in the proof of Lemma 3.4 we obtain the first estimate. Next, again from standard estimates, $\left\|\nabla \theta_{i}\right\|_{L^{2}\left(K_{i}^{\varepsilon}\right)} \leq c\left\|\theta_{i}\right\|_{H^{1 / 2}\left(\partial K_{i}^{\varepsilon} \backslash \Gamma_{r}^{\varepsilon}\right)}$. Note that

$$
\left\|\theta_{i}\right\|_{H^{1 / 2}\left(\partial K_{i}^{\varepsilon} \backslash \Gamma_{r}^{\varepsilon}\right)} \leq h\|W\|_{H^{1 / 2}\left(\partial K_{i}^{\varepsilon} \backslash \Gamma_{r}^{\varepsilon}\right)}+h z .
$$

From the exponential decay properties of $W$, and trace and interpolation inequalities, we gather that $\|W\|_{H^{1 / 2}\left(\partial K_{i}^{\varepsilon} \backslash \Gamma_{r}^{\varepsilon}\right)} \leq c$. Thus, the result follows.

Theorem 3.7. Let $u^{\varepsilon}$ be the solution of (1.1), and $u_{h}^{\varepsilon} \in V_{h}^{\varepsilon}$ be the solution of (2.4). Assume further (3.1), and that the trace of $\partial u^{0} /\left.\partial y\right|_{\Omega_{s}}$ on $\Gamma$ belongs to $H^{2}(\Gamma)$, where $u^{0}$ solves (3.3). Then there exists a constant $c$ independent of $\varepsilon$ and $h$ such that

$$
\left\|u^{\varepsilon}-u_{h}^{\varepsilon}\right\|_{H^{1}\left(\Omega^{\varepsilon}\right)} \leq c\left(h+d^{3 / 2}+\varepsilon h^{-1 / 2}+\varepsilon^{3 / 2} h^{-1}\right) .
$$

Proof. We shall base our proof on Lemma 3.5 and the asymptotics for $u^{\varepsilon}$ and the functions in $V_{h}^{\varepsilon}$. Let $\Upsilon(\mathbf{x})=(y-\varepsilon W) \partial u^{0} / \partial y(x, h) \chi_{r}^{\varepsilon}$. Using the triangle inequality and (3.6), it follows that

$$
\begin{array}{r}
\left|u^{\varepsilon}-v_{h}\right|_{H^{1}\left(\Omega^{\varepsilon}\right)} \leq\left|u^{\varepsilon}-u^{0}-u^{1}+\Upsilon\right|_{H^{1}\left(\Omega^{\varepsilon}\right)}+\left|u^{0}+u^{1}+\Upsilon-v_{h}\right|_{H^{1}\left(\Omega^{\varepsilon}\right)} \\
\leq c d^{3 / 2}+\left|u^{0}+u^{1}+\Upsilon-v_{h}\right|_{H^{1}\left(\Omega^{\varepsilon}\right)}
\end{array}
$$

for all $v_{h} \in V_{h}^{\varepsilon}$. We choose $v_{h}$ to be $u_{I}(\mathbf{x})=\sum_{\mathbf{x}_{i} \in \mathcal{N}}\left[u_{0}\left(\mathbf{x}_{i}\right)+u_{1}\left(\mathbf{x}_{i}\right)\right] \lambda_{i}(\mathbf{x})$. Then, in $\Omega_{s}, u_{I}$ is piecewise bilinear, continuous, and interpolates $u^{0}+u^{1}$. Thus

$$
\left|u^{0}+u^{1}-u_{I}\right|_{H^{1}\left(\Omega_{s}\right)} \leq c h .
$$

In $\Omega_{r}^{\varepsilon}$,

$$
\begin{gathered}
u_{I}(\mathbf{x})=(d+y-\varepsilon W-\varepsilon z) I_{h}\left(\frac{\partial u^{0}}{\partial y}\right)(x)+R_{I}, \\
R_{I}(\mathbf{x})=\varepsilon h^{-1} \sum_{i=1}^{N} \frac{\partial u^{0}}{\partial y}\left(x_{i}, 0\right)\left[\theta_{i}(\mathbf{x})+r_{i}(\mathbf{x})\right],
\end{gathered}
$$

where $I_{h}\left(\partial u^{0} / \partial y\right)(\cdot)$ is the piecewise linear interpolant of $\partial u^{0} / \partial y(\cdot, 0)$ in $(0,1)$. Let $e_{I}(x, y)=\partial u^{0} / \partial y(x, h)-I_{h}\left(\partial u^{0} / \partial y\right)(x)$ be the interpolation error. We need the estimates [15,29].

$$
\left\|e_{I}\right\|_{L^{2}(0,1)}+h\left\|\frac{\partial e_{I}}{\partial x}\right\|_{L^{2}(0,1)}+h\left\|e_{I}\right\|_{L^{\infty}(0,1)}+h^{2}\left\|\frac{\partial e_{I}}{\partial x}\right\|_{L^{\infty}(0,1)} \leq c h^{2}\left\|\frac{\partial u^{0}}{\partial y}\right\|_{H^{2}(0,1)} .
$$

We compute

$\left|\Upsilon-u_{I}\right|_{H^{1}\left(\Omega_{r}^{\varepsilon}\right)} \leq\left|y e_{I}\right|_{H^{1}\left(\Omega_{r}^{\varepsilon}\right)}+\varepsilon\left|w e_{I}\right|_{H^{1}\left(\Omega_{r}^{\varepsilon}\right)}+|d-\varepsilon z|\left|I_{h}\left(\frac{\partial u^{0}}{\partial y}\right)\right|_{H^{1}\left(\Omega_{r}^{\varepsilon}\right)}+\left|R_{I}\right|_{H^{1}\left(\Omega_{r}^{\varepsilon}\right)}$. 
Estimating each term we have

$$
\begin{gathered}
\left|y e_{I}\right|_{H^{1}\left(\Omega_{r}^{\varepsilon}\right)}^{2} \leq d \int_{\Omega_{r}^{\varepsilon}}\left|\frac{\partial e_{I}}{\partial x}\right|^{2} d \mathbf{x}+\int_{\Omega_{r}^{\varepsilon}}\left|e_{I}\right|^{2} d \mathbf{x} \leq c d^{2} h^{2}+c d h^{4} \\
\left|w e_{I}\right|_{H^{1}\left(\Omega_{r}^{\varepsilon}\right)}^{2} \leq\left\|e_{I}\right\|_{L^{\infty}(0,1)}^{2}|w|_{H^{1}\left(\Omega_{r}^{\varepsilon}\right)}^{2}+\left\|\frac{\partial e_{I}}{\partial x}\right\|_{L^{\infty}(0,1)}^{2}|w|_{L^{2}\left(\Omega_{r}^{\varepsilon}\right)}^{2} \leq c \varepsilon^{-1} h^{2}+c \varepsilon \\
|d-\varepsilon z|^{2}\left|I_{h}\left(\frac{\partial u^{0}}{\partial y}\right)\right|_{H^{1}\left(\Omega_{r}^{\varepsilon}\right)}^{2} \leq c d^{2}\left|I_{h}\left(\frac{\partial u^{0}}{\partial y}\right)\right|_{H^{1}\left(\Omega_{r}^{\varepsilon}\right)}^{2} \leq c d^{2} \int_{\Omega_{r}^{\varepsilon}}\left|\frac{\partial I_{h}\left(\frac{\partial u^{0}}{\partial y}\right)}{\partial x}\right|^{2} d \mathbf{x} \leq c d^{3} .
\end{gathered}
$$

Using Lemma 3.6 we finally estimate

$$
\left|R_{I}\right|_{H^{1}\left(K_{i}^{\varepsilon}\right)}^{2} \leq c \varepsilon^{2} h^{-2}\left(\left\|\theta_{i}\right\|_{H^{1}\left(K_{i}^{\varepsilon}\right)}^{2}+\left\|r_{i}\right\|_{H^{1}\left(K_{i}^{\varepsilon}\right)}^{2}\right) \leq c\left(\varepsilon^{2}+\varepsilon^{3} h^{-1}\right) .
$$

Adding up over the elements we obtain $\left|R_{I}\right|_{H^{1}\left(K_{i}^{\varepsilon}\right)} \leq c\left(\varepsilon h^{-1 / 2}+\varepsilon^{3 / 2} h^{-1}\right)$. Finally, from (3.10) we gather that

$$
\left|\Upsilon-u_{I}\right|_{H^{1}\left(\Omega_{r}^{\varepsilon}\right)} \leq c\left(d h+d^{1 / 2} h^{2}+\varepsilon^{1 / 2} h+\varepsilon^{3 / 2}+d^{3 / 2}+\varepsilon h^{-1 / 2}+\varepsilon^{3 / 2} h^{-1}\right) .
$$

The theorem follows from (3.1), (3.8), (3.9), and (3.11).

Remark 3.8. If $d \leq c_{1} h^{2 / 3}$, and $\varepsilon \leq c_{2} h$ for some constants $c_{1}, c_{2}$, then

$$
\left\|u^{\varepsilon}-u_{h}^{\varepsilon}\right\|_{H^{1}\left(\Omega^{\varepsilon}\right)} \leq c h+c \varepsilon h^{-1 / 2} .
$$

In practice it is wise to choose $d$ as small as possible, reducing the computational costs associated with computing the basis functions $\lambda_{i}$.

In Theorem 3.7 and estimate (3.12), the $\varepsilon h^{-1 / 2}$ error is related to the choice of (linear) boundary conditions for the $\lambda_{i}$ which originates the troublesome term $\theta_{i}$ in (3.7). Of course, the exact solution is far from being linear close to the rough boundary, and such a mismatch indicates that indeed $\theta_{i}$ is spurious.

Such a type of resonance error is not an exclusivity of the present method. For instance, some multiscale methods (e.g. MsFEM, and Residual Free Bubbles) proposed to tackle PDEs with rapidly varying coefficients also suffer from the same malady, and various strategies were devised to overcome it [22, [23, 24], 30].

One promising strategy to avoid such boundary effects in our case is oversampling [19, 21,22, 24]. The goal is to avoid the restriction that $\lambda_{i}$ is linear over the edges. Consider the element $K_{i}^{\varepsilon}$, and the enlarged ficticious element

$$
\hat{K}_{i}^{\varepsilon}=\left\{(x, y) \in \Omega_{r}^{\varepsilon}: i h-l<x<(i+1) h+l\right\},
$$

for some $l>0$. Let the auxiliary multiscale functions $\psi_{i}, \psi_{i+1}$ be the solutions of (2.3) in $\hat{K}_{i}^{\varepsilon}$. We define then $\lambda_{i}$ as

$$
\lambda_{i}=c_{i}^{1} \psi_{i}+c_{i}^{2} \psi_{i+1},
$$

and the constants $c_{i}^{1}, c_{i}^{2}$ are uniquely determined from the restriction $\lambda_{i}\left(\mathbf{x}_{j}\right)=\delta_{i j}$ for $j=i, i+1$. The numerical solution is defined by (2.4), where again $V_{h}^{\varepsilon}=$ $\operatorname{span}\left\{\lambda_{i}\right\}$.

To conclude the definition of such an oversampling method, it is necessary to define $l$. Such a constant needs to be large enough so that the effect in $K_{i}^{\varepsilon}$ of the boundary condition on $\partial \hat{K}_{i}^{\varepsilon} \cap \Omega^{\varepsilon}$ is negligible. On the other hand, increasing $l$ augments computational costs. A good compromise would be $l=O(\varepsilon)$.

Note that the method becomes nonconforming as $\lambda_{i}$ might have jumps over the edges, and thus $V_{h}^{\varepsilon} \not \subset H^{1}\left(\Omega^{\varepsilon}\right)$ in general. 


\section{Conclusion}

In this work, we propose a multiscale finite element scheme to deal with PDEs posed in domains with rough boundary. Previous techniques approach this problem with homogenization techniques. As far as we now, the exceptions are [27]28], but their methods seem more complicated than ours.

The present method is quite general, and its definition does not assume any special feature of the wrinkles. Since the scheme is based on solutions of local problems, parallelization is trivial. Of course, the method is still expensive since local problems depend on $\varepsilon$, but it is much cheaper than using pure piecewise linear FEM in the whole domain. However, if the wrinkles are periodic, it is still cheaper to use traditional wall-laws.

Concerning estimates, an error analysis is available for the periodic case only, where models using wall-laws have the $H^{1}\left(\Omega^{\varepsilon}\right)$ norm error as $h+\varepsilon^{1 / 2}$, and the present method converges as $h+\varepsilon h^{-1 / 2}$. The $h^{-1 / 2}$ term is related to a resonance error, also present in other multiscale methods applied to PDEs with highly oscillatory coefficients [19,21,24,30. Oversampling techniques might ameliorate such a problem.

\section{REFERENCES}

[1] Toufic Abboud and Habib Ammari, Diffraction at a curved grating: approximation by an infinite plane grating, J. Math. Anal. Appl. 202 (1996), no. 3, 1076-1100. MR1408368 (98b:78028)

[2] Diffraction at a curved grating: TM and TE cases, homogenization, J. Math. Anal. Appl. 202 (1996), no. 3, 995-1026. MR1408364 (98b:78027)

[3] Yves Achdou, Olivier Pironneau, and Frédéric Valentin, Shape control versus boundary control, Équations aux dérivées partielles et applications, 1998, pp. 1-18. MR1648212 (99j:49080)

[4] Yves Achdou, O. Pironneau, and F. Valentin, Effective boundary conditions for laminar flows over periodic rough boundaries, J. Comput. Phys. 147 (1998), no. 1, 187-218. MR1657773 (99j:76086)

[5] Y. Achdou, P. Le Tallec, F. Valentin, and O. Pironneau, Constructing wall laws with domain decomposition or asymptotic expansion techniques, Comput. Methods Appl. Mech. Engrg. 151 (1998), no. 1-2, 215-232. MR1625432 (99h:76027)

[6] Yves Achdou and Olivier Pironneau, Domain decomposition and wall laws, C. R. Acad. Sci. Paris Sér. I Math. 320 (1995), no. 5, 541-547 (English, with English and French summaries). MR1322334 (95k:76087)

[7] Grégoire Allaire and Micol Amar, Boundary layer tails in periodic homogenization, ESAIM Control Optim. Calc. Var. 4 (1999), 209-243 (electronic) (English, with English and French summaries). MR 1696289 (2000k:35019)

[8] Youcef Amirat and Olivier Bodart, Numerical approximation of laminar flows over rough walls with sharp asperities, J. Comput. Appl. Math. 164/165 (2004), 25-38. MR2056866 (2004m:76118)

[9] Youcef Amirat, Blanca Climent, Enrique Fernández-Cara, and Jacques Simon, The Stokes equations with Fourier boundary conditions on a wall with asperities, Math. Methods Appl. Sci. 24 (2001), no. 5, 255-276. MR.1818895(2002b:76042)

[10] Y. Amirat, O. Bodart, U. De Maio, and A. Gaudiello, Asymptotic approximation of the solution of the Laplace equation in a domain with highly oscillating boundary, SIAM J. Math. Anal. 35 (2004), no. 6, 1598-1616 (electronic). MR2083791 (2005h:35064)

[11] Ivo Babuška, Gabriel Caloz, and John E. Osborn, Special finite element methods for a class of second order elliptic problems with rough coefficients, SIAM J. Numer. Anal. 31 (1994), no. 4, 945-981. MR.1286212 (95g:65146)

[12] Gabriel R. Barrenechea, Patrick Le Tallec, and Frédéric Valentin, New wall laws for the unsteady incompressible Navier-Stokes equations on rough domains, M2AN Math. Model. Numer. Anal. 36 (2002), no. 2, 177-203. MR1906814 (2003k:76104) 
[13] Arnaud Basson and David Gérard-Varet, Wall laws for fluid flows at a boundary with random roughness, ArXiv:math.AP/0606768v1 (29 Jun 2006).

[14] Didier Bresch and Vuk Milisic, Higher order boundary layer corrector and wall laws derivation: a unified approach, ArXiv:math.AP/0611083v1 (3 Nov 2006).

[15] Philippe G. Ciarlet, The finite element method for elliptic problems, North-Holland Publishing Co., Amsterdam, 1978. Studies in Mathematics and its Applications, Vol. 4. MR0520174 $(58: 25001)$

[16] Weinan E and Bjorn Engquist, Multiscale modeling and computation, Notices Amer. Math. Soc. 50 (2003), no. 9, 1062-1070. MR2002752 (2004m:65163)

[17] _ The heterogeneous multiscale methods, Commun. Math. Sci. 1 (2003), no. 1, 87-132. MR1979846 (2004b:35019)

[18] Weinan E and Ping-bing Ming, Analysis of multiscale methods, J. Comput. Math. 22 (2004), no. 2, 210-219. MR.2058933(2005d:65188)

[19] Yalchin R. Efendiev, Thomas Y. Hou, and Xiao-Hui Wu, Convergence of a nonconforming multiscale finite element method, SIAM J. Numer. Anal. 37 (2000), no. 3, 888-910 (electronic). MR.1740386 (2002a:65176)

[20] Yalchin Efendiev and Alexander Pankov, Numerical homogenization of monotone elliptic operators, Multiscale Model. Simul. 2 (2003), no. 1, 62-79 (electronic). MR2044957 (2005a:65153)

[21] Thomas Y. Hou, Numerical approximations to multiscale solutions in partial differential equations, Frontiers in numerical analysis (Durham, 2002), 2003, pp. 241-301. MR2006969 (2004m:65219)

[22] Thomas Y. Hou and Xiao-Hui Wu, A multiscale finite element method for elliptic problems in composite materials and porous media, J. Comput. Phys. 134 (1997), no. 1, 169-189. MR.1455261 (98e:73132)

[23] Thomas Y. Hou, Xiao-Hui Wu, and Zhiqiang Cai, Convergence of a multiscale finite element method for elliptic problems with rapidly oscillating coefficients, Math. Comp. 68 (1999), no. 227, 913-943. MR1642758 (99i:65126)

[24] Thomas Y. Hou, Xiao-Hui Wu, and Yu Zhang, Removing the cell resonance error in the multiscale finite element method via a Petrov-Galerkin formulation, Commun. Math. Sci. 2 (2004), no. 2, 185-205. MR2119937 (2005m:65268)

[25] Alexandre Madureira and Frédéric Valentin, Analysis of curvature influence on effective boundary conditions, C. R. Math. Acad. Sci. Paris 335 (2002), no. 5, 499-504 (English, with English and French summaries). MR.1937121 (2003j:35054)

[26] Alexandre L. Madureira and Frédéric Valentin, Asymptotics of the Poisson problem in domains with curved rough boundaries, SIAM J. Math. Anal. 38 (2006/07), no. 5, 1450-1473 (electronic). MR 2286014 (2007j:35031)

[27] Serge Nicaise and Stefan A. Sauter, Efficient numerical solution of Neumann problems on complicated domains, Calcolo 43 (2006), no. 2, 95-120. MR2245226 (2007c:65108)

[28] M. Rech, S. Sauter, and A. Smolianski, Two-scale composite finite element method for Dirichlet problems on complicated domains, Numer. Math. 102 (2006), no. 4, 681-708. MR2207285 (2006m:65283)

[29] H.-G. Roos, M. Stynes, and L. Tobiska, Numerical methods for singularly perturbed differential equations, Springer Series in Computational Mathematics, vol. 24, Springer-Verlag, Berlin, 1996. Convection-diffusion and flow problems. MR1477665 (99a:65134)

[30] Giancarlo Sangalli, Capturing small scales in elliptic problems using a residual-free bubbles finite element method, Multiscale Model. Simul. 1 (2003), no. 3, 485-503 (electronic). MR2030161 (2004m:65202)

[31] Marcus Sarkis, Private Communication, 2007.

CoordenaÇão de Matemática Aplicada e Computacional, Laboratório Nacional de Computação Científica, Av. Getúlio Vargas 333, CeP 25651-070 Petrópolis - RJ, Brazil

E-mail address: alm@lncc.br 